\title{
ESTUDOS PROSPECTIVOS E A ORGANIZAÇÃO DE SISTEMAS DE INOVAÇÃO NO BRASIL
}

\author{
Mauro Zackiewicz \\ Maria Beatriz Bonacelli \\ Sergio Salles Filho
}

\begin{abstract}
Resumo: As condições institucionais para a competitividade na sociedade do conhecimento são dinâmicas e estão em contínua reconstrução, sendo a capacidade de inovação tecnológica um de seus fatores determinantes. O elevado grau de incerteza e o alto custo associados à inovação tecnológica são desafios para a sustentação dos padrões tradicionais de investimento em ciência e tecnologia. Este artigo centra-se no tema do desenvolvimento de capacidade antecipativa e a incorporação da função prospectiva na gestão das organizações de C,T\&I.

Palavras-chave: Políticas de C,T\&I. Prospectiva tecnológica. Inovação tecnológica.

Abstract: Competitiveness in the knowledge society is more and more dependent on fostering innovative activities. The high degrees of incertitude and the increasing costs associated to technological development are challenging the present levels of investment in S,T\&I. This article focuses on this theme specially on the necessity of developing anticipative capabilities in order to create a prospective culture in S,T\&I organizations. Key words: Science and Technology policies. Technological prospective. Technological innovation.
\end{abstract}

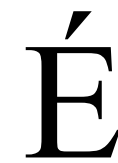

notável a crescente importância dos estudos prospectivos. Hoje, eles são componentes fundamentais para políticas e estratégias de inovação, não só como subsídios para ampliar a capacidade de antecipação, mas também porque estimulariam virtuosamente a organização dos sistemas de inovação.

Este artigo propõe-se a discutir, sob o ponto de vista do conceito de sistema de inovação, alguns dos meios pelos quais tal efeito estruturante poderia se manifestar a partir da execução de estudos prospectivos.

Em sua origem, os estudos prospectivos para os rumos da ciência e da tecnologia buscavam aumentar ao máximo a capacidade de previsão de seus avanços - algo plausível no contexto de estabilidade institucional que caracterizou as décadas de 50 e 60 . A partir dos centros de pesquisa militares dos EUA motivados pelo conflito velado da Guerra Fria, inúmeras técnicas e metodologias foram criadas e aperfeiçoadas para prever o desenvolvimento tecnológico do inimigo e orientar as estratégias da P\&D militar. Empresas e outros setores da sociedade civil rapidamente se apropriaram de muitas dessas ferramentas e as incorporaram a suas rotinas de planejamento e de busca por oportunidades.

Uma segunda corrente de estudos prospectivos desenvolveu-se a partir da década de 70 , em contrapartida à tendência tecnocentrada predominante: a dos futuristas (JOHNSTON, 2002). Sua abordagem dava ênfase à compreensão das raízes profundas e da evolução dos propósitos da humanidade, de seus valores, condições e expectativas.

Quando ficou mais claro que a estabilidade era a exceção e que a mudança técnica exercia uma influência mais complexa do que se supunha nas mudanças institucionais, das quais também era dificilmente dissociável, a quimera da previsão tecnológica e a utopia normativa dos futuris- 
tas foram enfraquecidas e amalgamadas em abordagens bem mais pragmáticas. Assim, é comum encontrar definições para prospecção tecnológica como:

A systematic attempt to look into the longer-term future of science, technology, economy and society with a view to identifying emerging generic technologies likely to yield the greatest economic and social benefits (OCDE, 1996).

A percepção de que o fenômeno da inovação tecnológica é complexo e se manifesta a partir de diversos níveis, em processos multicausais e retroalimentados e com a atuação mais ou menos determinante de diversos atores, trouxe a necessidade de projetá-lo sobre a sintaxe dos sistemas abertos. Se, por um lado, pensar a inovação a partir de sistemas superava a simplicidade arriscada dos antigos modelos lineares, de inovação, por outro, criava novas dificuldades analíticas - especialmente para a identificação das relações causais responsáveis pelas novas tecnologias e para as projeções prospectivas.

\section{COMPLEXIDADE, INCERTEZA E AMBIGÜIDADE}

A partir da década de 80, diversos autores perceberam que a mudança para uma óptica sistêmica na explicação do fenômeno da inovação implicava, também, em mudanças nos métodos e propósitos da prospecção tecnológica (MARTIN; IRVINE, 1989; GODET, 1993). Desde então, foram feitas diversas tentativas, propostas novas metodologias e cunhados novos termos.

Paul David (2002) faz uma interessante crítica aos estudos recentes de technology foresight no Reino Unido, dizendo que eles não superaram totalmente o antigo modelo linear, o que ocorreu foi muito mais uma inversão. David afirma que a exagerada ênfase na orientação pelo mercado (demand-pull) pode ser desastrosa e que seria simplista tentar enquadrar o avanço - e o financiamento público - da ciência e tecnologia apenas por demandas de curto prazo e de alto retorno econômico potencial. Além disso, com a insistência nesse processo, poderiam ocorrer mudanças organizacionais nas instituições de $\mathrm{C} \& \mathrm{~T}$ que, ao invés de gerar o círculo virtuoso da inovação, levariam a uma perda sistêmica de eficiência na produção de novos conhecimentos. O autor prefere chamar de "ciência aberta" o que comumente se chama de "ciência básica" e advoga a manutenção do investimento público como o mais adequado para financiar a produção da commodity "conhecimento", assumindo seu caráter incerto e aleató- rio. Entretanto, não descarta a validade de políticas voltadas para a inovação que definam linhas de ação e pesquisa prioritárias - desde que não sufoquem o espaço da ciência aberta. Uma vez que os recursos são limitados, elas passariam por reconfigurações de papéis institucionais e culturas organizacionais que ainda não se materializaram na prática. $\mathrm{O}$ fato é que é difícil criar instrumentos de política, planejamento e avaliação que estejam plenamente de acordo com a noção de sistema de inovação e estabeleçam um grau de coerência e determinação compatível com os dos modelos lineares, que são simplistas, porém facilmente comunicáveis. A maior parte dos atores envolvidos nessas atividades ainda tem dificuldade de operar em um marco cognitivo que estabeleça a complexidade, a incerteza e a ambigüidade como regra.

Os grandes desafios metodológicos da atualidade para os estudos prospectivos referem-se a essas questões. A política para a organização de sistemas de inovação não pode se restringir a incorporar o lado da demanda nem a transferir todo o ônus do financiamento para o setor privado. A política precisa, essencialmente, promover ações que levem a um novo patamar de entendimento do papel da C\&T na sociedade - e os estudos prospectivos são decisivos nesse processo. Afinal, a noção de prospecção quase se confunde com esse objetivo.

Tanto, que a tendência atual dos estudos prospectivos é a de evoluir das abordagens estritamente tecnológicas para outras que enfatizem questões organizacionais do desenvolvimento da C\&T no contexto de sistemas de inovação (RENN, 2002). Essa tendência também aproxima os estudos prospectivos dos sistemas de apoio à decisão para o planejamento e dos esforços de avaliação da evolução das redes sociotécnicas (ZACKIEWICZ, 2002). Cada vez mais, os estudos sobre o futuro das atividades de $C \& T$ deixam de ser encarados como encomendas ad hoc para se tornarem atividades perenes e internalizadas nas organizações presentes nos sistemas de inovação. Os estudos prospectivos deparam-se com o desafio da complexidade a partir do momento em que há dificuldade para estabelecer os nexos causais para deduzir as múltiplas possibilidades de mudanças técnicas e suas conseqüências (RENN, 2002).

A incerteza é relacionada com a complexidade, mas se distingue desta por se referir ao grau de confiança nas relações causais estabelecidas por um modelo explicativo, e não ao modelo em si. A incerteza aumenta se a complexidade não puder ser resolvida por teorias e métodos que gerem modelos mais adequados. Se o efeito estudado possuir natureza estocástica, isso também colaborará para a incerteza (RENN, 2002). 
A ambigüidade refere-se à variabilidade das interpretações possíveis - e legitimadas teoricamente - a partir de uma mesma base de dados ou a partir da observação dos mesmos fatos. Alta complexidade e incerteza favorecem o surgimento de controvérsias e, portanto, de ambigüidade (RENN, 2002).

A emergência desses atributos no contexto dos estudos sobre a evolução dos processos de inovação é argumento tanto para a apologia quanto para a crítica sobre a necessidade de planejamento e de definição de prioridades. De um lado, os que defendem o enquadramento dos rumos da C\&T apoiados na execução de estudos prospectivos advogam que, desse modo, seria possível reduzir a incerteza e a ambigüidade na execução das pesquisas, além de melhor orientá-las para as necessidades econômicas e sociais. De outro lado, argumenta-se que, por conta da complexidade e da imprevisibilidade dos processos de inovação, todo planejamento ancorado em metodologias prospectivas seria claramente reducionista e nocivo à riqueza de possibilidades que o avanço do conhecimento poderia proporcionar, se fosse estimulado em toda sua potencialidade.

Novamente, o que está em jogo é a questão da organização. Seria possível conjugar o melhor dos dois lados? Seria possível fazer com que o planejamento e a definição de prioridades orientadas a problemas do país potencializassem ainda mais o avanço do conhecimento?

Para tentar uma resposta afirmativa a essa questão, é preciso examinar como a prospecção pode estimular a organização dos sistemas de inovação e o surgimento de novos padrões cognitivos acerca da produção de conhecimento e tecnologia.

\section{EFEITOS SOBRE A ORGANIZAÇÃO DA INOVAÇÃO}

A idéia de que os resultados da prospecção tecnológica são úteis para a organização dos processos de inovação é tributária da escola norte-americana do pós-guerra e foi amplamente disseminada, junto com suas metodologias e abordagens, principalmente por autores interessados nas estratégias e no planejamento empresarial.

Com o surgimento do conceito de Sistemas Nacionais de Inovação - SNIs na década de 80 (FREEMAN, 1987), essa idéia ganha em escopo ao identificar uma série de efeitos colaterais dos processos subjacentes à obtenção dos resultados prospectivos que atuariam como fatores estruturantes para os SNIs.
A partir de então, a ênfase voltou-se fortemente para os processos, muitas vezes até em detrimento dos produtos. Métodos como o Delphi ganharam nova força sob o novo enfoque. Diversos estudos - agora também chamados exercícios - prospectivos foram lançados pelo mundo para estruturar e definir prioridades a seus SNIs. ${ }^{2}$ Irvine e Martin (1984), bem ao estilo minimalístico das ferramentas de gestão, enxergam a essência desse novo espírito nos 5Cs do technology foresight: concentração, comunicação, consenso, compromisso e coordenação. A idéia de concentração refere-se ao fato de que os participantes de um exercício de prospecção são convidados a refletir de modo sistemático sobre certas questões, definir prioridades e buscar identificar a relevância de suas próprias atividades. Comunicação, porque os exercícios prospectivos se transformariam em canais por onde a informação fluiria de modo eficiente e plural. O consenso é um atributo buscado explicitamente e é o sustentáculo da identificação e da seleção de prioridades de investimento a partir da prospecção. Dado o caráter participativo e "socialmente construído" dos processos de foresight, o compromisso dos atores envolvidos seria mais um resultado, a garantir a legitimidade do processo e viabilizar a efetiva implementação das prioridades estabelecidas. Por fim, a coordenação do sistema de inovação e a cooperação na pesquisa seriam promovidas pela formação de redes proporcionada pelas conexões entre pessoas, grupos e temas de pesquisa derivados do exercício.

Claramente, os 5Cs não são independentes entre si. Aparecem acima listados em uma ordem causal na qual cada $\mathrm{C}$ seria uma etapa rumo à coordenação. $\mathrm{O}$ sucesso de cada etapa seria o impulso para a seguinte. Porém, esse processo é fortemente condicionado pela metodologia adotada, por contextos políticos e institucionais do país em que ocorre o exercício e pelo entendimento prévio dos atores em relação aos objetivos do estudo prospectivo.

De fato, trata-se de um processo de aprendizado que poderia levar a uma reorganização mais ampla do que esses 5Cs mostram - especialmente no contexto dos países em desenvolvimento. Isso é importante porque coloca outras questões que precisam ser observadas.

A questão do aprendizado está presente nos dois níveis nos quais os estudos prospectivos atuam - ou seja, tanto na capacidade de antecipação quanto na organização da inovação. No primeiro deles, o aprendizado refere-se à construção progressiva de noções mais ou menos 
compartilhadas sobre como será o futuro da sociedade e as implicações das tecnologias que poderão surgir e/ou se tornar dominantes em determinado campo. São essas visões que fundamentam as decisões dos atores no presente e, de certo modo, moldam as trajetórias tecnológicas. Idier (2000) chama a atenção para o componente motivacional da inovação e observa o quanto as imagens socialmente compartilhadas sobre o futuro incluindo aí fatores culturais, psicológicos e estéticos ${ }^{3}$ - influenciariam as decisões e, portanto, as trajetórias tecnológicas. Assim, a atividade prospectiva teria um papel mais profundo do que aquilo que Irvine e Martin chamam de "concentração" e "comunicação". A natureza compartimentalizada do conhecimento organizado em disciplinas e especialidades estanques, a lógica reativa e de curto prazo de grande parte dos atores das cadeias produtivas, as múltiplas influências culturais e heranças institucionais, tudo isso cria obstáculos formidáveis à concentração e à comunicação. Eles começam nas diferenças semânticas e se aprofundam em direção às diferenças de valores e expectativas. Esses obstáculos só podem ser superados por meio de aprendizado, mas a geração de círculos virtuosos de aprendizado que culminem em frentes de consenso - o próximo passo dos $5 \mathrm{Cs}$ - não é direta e nem tampouco óbvia.

A abordagem invariavelmente adotada para viabilizar esse salto é a da participação. Essa idéia já é, por princípio um tanto nebulosa, e mais facilmente apropriada pela retórica do que pela ação. Entretanto, é na prática da participação que está o segundo nível de aprendizado, o que desde logo mais interessa à organização da inovação e que, não obstante, é ainda o mais incipiente e fragilmente compreendido.

Antes de colaborar para a superação dos obstáculos impostos à concentração e à comunicação, a participação também é dificultada e limitada pelos mesmos obstáculos. Assim, se nas oportunidades que a participação for invocada, a discussão for orientada para objetos que reforcem esses obstáculos, o resultado poderá ser o inverso do esperado. A participação precisa ocorrer sobre enfoques multidisciplinares, sobre visões conflitantes, sobre referências multiculturais. A participação precisa exigir o aprendizado dos atores, a compreensão dos limites impostos pelos contextos institucionais nos quais estão inseridos e a ampliação de suas visões particulares de futuro. Renn (2002) atribui ainda à participação a função de reduzir a ambigüidade e os custos de transação entre os atores, uma vez que a busca por frentes de consenso evitaria a maximização parcial dos benefícios dos atores.

É na reação à ação desconstrutiva da participação que está a chave do aprendizado para uma organização mais dinâmica da inovação. A formação de redes, a definição de competências essenciais, o compromisso com prioridades, o compromisso público, são todos características de um certo padrão de organização fundamentado em formas comportamentais e contratuais que precisam ser, antes de tudo, aprendidas. Essas novas formas de organização cresceriam então no espaço vazio do futuro, de modo tão aberto à criatividade quanto as tecnologias e o conhecimento que produziriam.

A coordenação a que Irvine e Martin fazem referência envolve esses atributos. Mas fica claro que essa abordagem percebe mais o dinamismo das tecnologias que a necessidade do dinamismo das organizações e instituições. Como bem observou Johnston (2002) ao analisar os principais exercícios de prospecção no cenário internacional, não há conexão natural entre prospecção, planejamento e tomada de decisão. E tampouco há, necessariamente, o efeito virtuoso de coordenação sobre o sistema de inovação. Na prática, as abordagens metodológicas têm dificuldade para concretizar esses saltos. Isso reforça a importância de se ampliar o entendimento conceitual do que significa, hoje, fazer prospecção tecnológica, para que se reformulem as abordagens metodológicas vigentes que, em sua esmagadora maioria, apenas tangenciam as questões realmente importantes para a política e a gestão da inovação.

\section{ESBOÇO PARA UM ESQUEMA ANALÍTICO}

Nessa seção, é proposta uma primeira tentativa de construção de um esquema analítico para estudos prospectivos. O objetivo é duplo: caracterizar os estudos de modo a permitir a identificação de correlações entre objetivos, padrões metodológicos, tipos de organizações envolvidas como atores e, finalmente, as áreas e formas de conhecimento consideradas; e medir o impacto dos estudos prospectivos na organização da inovação.

Por meio dessa análise, espera-se aprofundar o entendimento dos nexos conceituais e metodológicos da função estruturante da prospecção tecnológica, assim como fornecer indicadores que permitam a seus executores melhor orientá-la nesse sentido.

Desse modo, para a caracterização dos estudos prospectivos, não obstante a já clássica tipologia propos- 
ta por Martin e Irvine (1989), há especial interesse em certos aspectos qualificáveis e quantificáveis dos exercícios prospectivos. O esquema analítico considera importante a associação de escalas de medida às variáveis consideradas, para permitir a identificação de correlações e padrões que poderão gerar conclusões interessantes.

Assim, para a caracterização dos exercícios prospectivos, consideram-se quatro variáveis:

- A aderência do objetivo do exercício prospectivo aos seguintes objetivos gerais e não excludentes (escala nenhuma; implícita; marginal; explícita): a) prospecção de áreas de conhecimento e tecnologias emergentes; b) identificação de tecnologias críticas para cadeias produtivas; c) prospecção voltada para problemas sociais ou ambientais; d) identificação de competências essenciais; e) definição de prioridades; f) formação de redes de pesquisa voltadas para problemas.

- As técnicas utilizadas para compor a metodologia (escala - forma clássica; modificada; associada a $x$, associada a $y$, etc. ${ }^{4}$ ): a) Delphi; b) cenários; c) painéis de atores (plataformas); d) extrapolação de séries temporais; e) modelos de simulação dinâmica; f) análise morfológica; g) métodos multicritérios; h) mineração de dados ou textual; i) evolução de mapas de conhecimento e monitoramento tecnológico.

- A estrutura institucional do estudo se dá entre os seguintes atores (escala - não participam; quantos indivíduos têm participação passiva; quantos indivíduos têm participação ativa; quantos indivíduos podem ser considerados decisores): a) empresas; b) universidades; c) institutos de pesquisa; d) organizações da sociedade civil; e) agências regionais de fomento e planejamento; f) agências nacionais de fomento e planejamento; g) agências supranacionais de fomento e planejamento; h) mídia; i) indivíduos sem vínculo formal com as organizações anteriores.

- A estrutura cognitiva do estudo refere-se a (escala - não se refere; refere-se um pouco; refere-se bastante; referese quase/ou exclusivamente): a) demandas dos usuários; b) gargalos de cadeias produtivas; c) problemas sociais ou ambientais; d) condicionantes do futuro e causalidades; e) projetos ou programas científicos ou tecnológicos; f) disciplinas; g) técnicas ou tecnologias gerais; h) profissões.

Para medir os impactos sobre a organização da inovação é importante, antes, fazer a distinção entre os conceitos de impacto e de resultado. "Resultados" são os produtos diretos do exercício prospectivo, palpável e quantificáveis no tempo e no espaço: visões de futuro, previsões, prioridades, encontros e discussões. Esses objetos em si não interessam diretamente para este esquema analítico. Os "impactos" vêm depois dos resultados: são os efeitos que a existência desses objetos provocam em outras estruturas. Para a análise proposta, o que interessa são as transformações que ocorrem em estruturas-chave para a organização da inovação.

Desse modo, foram escolhidos três importantes elementos para descrever tal processo: o efeito sobre a coordenação entre os atores, o efeito sobre o aprendizado e o efeito sobre a evolução da estrutura do conhecimento. Para cada um desses impactos, foram selecionados três indicadores mensuráveis qualitativamente ou quantitativamente:

- Efeitos sobre a coordenação entre atores: a) intercâmbio de recursos humanos (mostrando a existência de agendas comuns ou complementares); b) redução dos custos de transação para a difusão de tecnologia e circulação de conhecimento; c) aumento e perenidade dos investimentos para pesquisas a longo prazo.

- Efeitos sobre o aprendizado: a) institucionalização da função prospectiva nas organizações; b) aumento nos registros de patentes e publicações em temas prioritários; c) novos arranjos organizacionais para a produção de conhecimento e tecnologia.

- Efeitos sobre a estrutura do conhecimento: a) aumento do número de projetos de pesquisa inseridos em contextos de aplicação; b) aumento de projetos de pesquisa e de publicações conduzidos em redes multiinstitucionais; c) aumento do número de cursos multidisciplinares.

Essa estrutura de impactos pode ser modelada de modo hierárquico e agregada a partir de funções de valor aditivas. Um modelo dessa natureza requer ainda alguns parâmetros auxiliares para uma interpretação mais realista do impacto de cada estudo prospectivo. Entre eles, coeficientes de ponderação da importância de cada indicador e efeito e coeficientes de credibilidade para cada indicador - uma vez que nem sempre a existência do impacto pode ser integralmente atribuída à existência de um exercício em particular.

Como foi destacado no início desta seção, esta proposta analítica ainda é fruto de um esforço inicial de construção. A abordagem que se pretende seguir é a de ampliar o entendimento estrutural e funcional dos exercícios de prospecção em sua relação com a organização dos siste- 
mas de inovação, mais do que a de determinar um conjunto de quesitos para julgá-los ex post em sua efetividade, embora isso também seja possível. A característica processual e perene que cada vez mais se imprime às atividades prospectivas exige que sejam criados mecanismos de ajuste e atualização, uma vez que um indicador de sucesso é a própria mudança do contexto institucional em que elas ocorrem. O tipo de análise que este artigo propõe vai nessa direção.

\section{CONCLUSÕES}

Johnston (2002) assinala que é difícil generalizar a experiência de prospecção dos diferentes países, que os países em desenvolvimento tendem a mimetizar os exercícios dos desenvolvidos e que por isso não são tão bemsucedidos. Acrescenta que é preciso metodologias e abordagens próprias. É certo que há uma concepção de procedimentos que têm aplicação geral, mas não é menos certo que as distintas realidades de países, regiões e organizações contribuem para a definição de metodologias específicas.

Em qualquer ambiente complexo, o velho e o novo não se dissociam, as percepções e interpretações tributárias a ambos convivem lado a lado, por vezes durante longo tempo. Dependendo do quadro institucional vigente, ambigüidade e incerteza assumem dimensões particulares. E esse é um bom ponto de partida quando se pretende instituir a prática do planejamento e da prospecção. Organizar sistemas de inovação significa mexer com ambientes complexos. É tarefa de longo prazo que combina atores diferentes e suas diferentes perspectivas. Mais que isso: é tarefa balizada por alvos em constante movimento. É, portanto, uma atividade permanente. Os princípios do foresight (os 5Cs) são especialmente importantes, em todos os níveis: macro, meso e microinstitucionais. Criar uma cultura que incorpore esses princípios especialmente uma cultura voltada à formação de canais de comunicação, de planejamento participativo entre os diversos atores dos sistemas de inovação -, é o desafio maior da estruturação da política de inovação, seja no nível microinstitucional (organizações de pesquisa, firmas, etc.), seja no mesoinstitucional (arranjos produtivos locais, sistemas locais), seja ainda no plano macroinstitucional (sistema nacional de inovação e seus componentes no âmbito federal).

\section{NOTAS}

Este artigo faz parte da pesquisa Grupo de Estudos sobre Organização da Pesquisa e da Inovação - Geopi do Departamento de Política Científica e Tecnológica - DPCT/IG da Unicamp com financiamento da Finep.

1. Cf. Whitehill (1996) para uma breve revisão da evolução dos conceitos e para conhecer os autores mais importantes que adotaram essa perspectiva.

2. Uma revisão bastante completa sobre essa febre prospectiva pode ser realizada a partir de OCDE (1996), Gavigan e Scapolo (1999) e Johnston (2002).

3. Para o autor, as obras de ficção científica exerceriam uma forte influência na percepção social sobre o futuro das tecnologias. Cita o caso do projeto Guerra nas Estrelas do governo Reagan nos EUA e as previsões sombrias atribuídas à biotecnologia. A engenhosidade terrorista de 2001 pode também ter sido inspirada pela ficção.

4. As variáveis internas $x, y$, etc. referem-se às demais técnicas em suas formas clássicas e podem assumir valores de 0 a 9 . O comprimento do vetor de dados será definido pelo caso que contar mais associações na amostra considerada.

\section{REFERÊNCIAS BIBLIOGRÁFICAS}

BARRÉ, R. Foresights and Their Themes: Analysis, Typology and Perspectives. In: THE ROLE OF FORESIGHT IN THE SELECTION OF RESEARCH POLICY PRIORITIES.

Conference Papers... Seville, 13-14 May 2002. p. 88-109.

BARROS, H.G. Brazilian National Technology Foresight: The Prospectar Project. In: WALCUE SEMINAR, Paper, Cartagena de Indias, Colombia, January 22, 2002.

DAVID, P. The Political Economy of Public Science: a Contribution to the Regulation of Science and Technology. In: SMITH, H.L. (Ed.). The Regulation of Science and Technology. London: Palgrave Publishers, 2002.

FREEMAN, C. Technology policy and economic performance: lessons from Japan. London: Pinter Publishers, 1987.

GAVIGAN, J.P.; SCAPOLO, F. Matching Methods to the Mission: a comparison of national foresight exercises. Foresight, v. 1, n. 6, p. 491-513, 1999.

GODET, M. From Antecipation to Action: A Handbook of Strategic Prospective. Paris: Unesco, 1993.

IDIER, D. Science fiction and technology scenarios: comparing Asimov's robots and Gibson's cyberspace. Technology in Society, n. 22 , p. $255-272,2000$.

IRVINE, J.; MARTIN, B.R. Foresight in Science, Picking the Winners. London: Printer Publishers, 1984.

JOHNSTON, R. The State and Contribution of International Foresight: New Challenges In: THE ROLE OF FORESIGHT IN THE SELECTION OF RESEARCH POLICY PRIORITIES. Conference Papers... Seville, 13-14 May 2002. p. 59-74. 
MARTIN, B.R.; IRVINE, J. Research Foresight - Priority in Science. London: Pinter Publishers, 1989.

OCDE. Special Issue on Government Technology Foresight Exercises. STI Rewiew, Paris, n. 17, 1996.

RENN, O. Foresight and Multi-Level Governance. In: THE ROLE OF FORESIGHT IN THE SELECTION OF RESEARCH POLICY PRIORITIES. Conference Papers... Seville, 13-14 May 2002. p. 35-42.

WHITEHILL, M. Strategy Foresight: The Future of Strategy Research. Long Range Planning. v. 29, n. 2, p. 249-254, 1996.

ZACKIEWICZ, M. Em busca da inovação: as atuais perspectivas do estudo do futuro e da avaliação em ciência e tecnologia. Parcerias Estratégicas, CEE/MCT, n. 15, 2002.
Mauro Zackiewicz: Pesquisador Associado do Grupo de Estudos sobre Organização da Pesquisa e da Inovação - Geopi, Departamento de Politica Científica e Tecnológica da Unicamp (maurozac@ige.unicamp.br).

Maria Beatriz Bonacelli: Professora Doutora do Departamento. de Política Científica e Tecnológica da Unicamp (bia@ige.unicamp.br).

Sergio Salles Filho: Professor Titular do Departamento de Politica Científica e Tecnológica da Unicamp (sallesfi@ige.unicamp.br).

Artigo recebido em 18 de fevereiro de 2005.

Aprovado em 22 de março de 2005. 